\title{
Planning and Scheduling of an Agile Earth Observing Satellite Combining on-Ground and on-Board Decisions
}

\author{
Z.X. Chang, J.F. Li \\ College of Information Systems \& Management \\ National University of Defense Technology \\ Changsha, China
}

\begin{abstract}
The new generation of agile satellites has more freedom and choices on when and how to make an observation. That may lead to the needs of uniform and deliberate considering operating status and rules of on-board instruments in the management and control process. What's more, needs are improving responsiveness to dynamic environments, and lightening communication loads between the satellite and the ground. Given the restrictions on on-board computing ability, we propose a framework for integrated planning and scheduling of an agile satellite which combines both on-board and on-ground decisions. Different decision functions are divided and deployed. Simulative application in a corporation which is developing agile satellites shows that the planning and scheduling framework proposed in this paper is able to effectively support the management and control task of an agile satellite and adequately make use of its multiplication advantages in data acquirement.
\end{abstract}

Keywords-agile satellite; autonomous; earth observing; planning; scheduling

\section{INTRODUCTION}

If the satellite itself has a very small range of operations it can perform or if the estimation errors of satellite status and environment do not matter, the traditional way of task management is a reasonable option. However, earth observing satellites are becoming more and more capable. The new generation of agile earth observing satellite (AEOS) can move its bore sight along roll, pitch, or yaw axis at a faster speed. That will enable it to observe a specific ground target from theoretically numerous view angles corresponding to different positions and ephemeris times. Thus an AEOS does have many chances to avoid cloud or other adverse effects by choosing appropriate opportunities [1].

On-board autonomy can certainly help an AEOS to perform better by scheduling tasks at the right moment, considering weather and actual situation. This also would benefit an AEOS by improving the quantity of taken images and shortening user's waiting time .

The AEOS' primarily consideration in this paper has a capability to change its attitude fast along pitch and roll axis across large angle. We propose a framework to distribute planning and scheduling functions requiring different computing capabilities among the satellite and the ground management department. Afterwards, the on-board part will make on-line decision to repair the initial plan in condition of finding big deviations from the expected situations, which would cause the initial plan to become infeasible. We also explain the planning and scheduling algorithms in some detail. The framework and algorithms have been verified in a simulation-based satellite design case.

\section{The Planning And Scheduling Problem}

Planning and scheduling of earth observing satellite is defined here as: based on comprehensive considerations of satellite abilities and observing requirements, assign satellite resources to multiple tasks in contest, and determine specific activities and their begin and end times to fulfill the selected tasks, with an aim to eliminate conflicts and maximally satisfy users' requirements.

For an AEOS, its rolling ability enables the satellite to observe wider areas at both sides of its ground track, while pitching ability enables it to observe a ground target in a much longer time window. So the solar panel will also change its direction, rather than always have the opportunity to get largest amount of electric energy charged.

Thus planning and scheduling of the AEOS must explicitly consider energy and attitude factors, which means all operations consuming or generating energy or changing satellite attitude must be deliberately taken into account in a synchronized way when making a plan [3].

More details of the factors considered are discussed in the following.

\section{A. Planning and Scheduling Process}

Step 1: beforehand processing.

Step 2: integrated planning and scheduling.

Step 3: dynamic re-planning.

Step 4: command generation.

\section{THE DECISION FRAMEWORK}

To distribute some planning and scheduling function on board, it is necessary that the satellite be of certain autonomy.

There are some typical ways to realize a certain degree of autonomy that will be discussed first as follows. 


\section{A. Candidate Modes for Autonomy}

According to the degree of autonomy, management and control modes of a spacecraft can be mainly divided into five kinds from low level to high level.

\section{B. Combinatorial Decision Framework}

Taken together, we propose a planning and scheduling framework combining on-board and on-ground decision in reference of the mode 4 . It is particularly suitable for our case since the computing ability on board is strictly limited while the agility and communication restriction do require certain degree of autonomy. Our framework can be demonstrated as Figure 1.

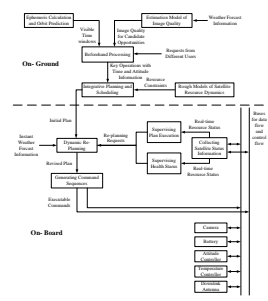

FIGURE I. THE COMBINATORIAL DECISION FRAMEWORK FOR PLANNING AND SCHEDULING.

\section{Planning And Scheduling Algorithm On GROUnd}

Due to the mixed planning and scheduling features and many dynamics needed to be estimated and considered, it is really hard to model the problem as some usual formats such as mixed integer programming and then use sophisticated methods to solve it. To get through these difficulties, we use some rule-based heuristics that are efficient for the problem.

\section{A. Primary Work-Flow of the Algorithm}

The algorithm starts from time point 0 which is the beginning of a planning horizon and goes ahead along the timeline while using some rules for decision. All the opportunities for observation and data downloading are ordered according to their earliest start time. So we get a queue of key operations with the first one as a current operation to be considered. Primary flow of the algorithm can be demonstrated in Figure 2.

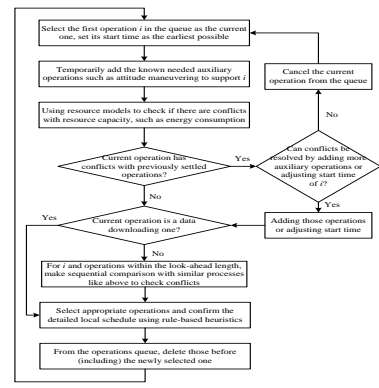

FIGURE II. PRIMARY WORK-FLOW FOR THE PLANNING AND SCHEDULING ALGORITHM.

\section{B. The Rule-Based Heuristics}

\section{About Look-Ahead Length}

Look-ahead length is just the number of key operations in the queue. We set a maximal length as a constant integer more than 0 . But it is not fixed, since for data downloading operations, looking ahead is not needed, equivalent to a length of 0 .

\section{D.About Start Time of Observation}

As mentioned, an observing opportunity for an AEOS is much longer than the observation needs. The algorithm always tries to schedule an imaging operation as early as possible with an acceptable estimated image quality. This will intuitionally reduce the possibility of conflicts with behind operations in the queue. The implied intention is to avoid frequent retracts and repeated adjustments.

\section{E. About Memory Erasing}

Memory erasing is a relative independent operation. According to management feature of the satellite, we set an upper threshold value as a certain percent of the maximal storage capacity. If data accumulates to catch that threshold, a memory erasing operation is triggered.

\section{F. About Data Download Order}

Data recorded in storage is in a form of files, which is ordered by revenues of corresponding customer requests. For a specific data downloading operation, data will be downloaded in that order considering available time. It is required that one file must be downloaded as a whole to one ground station. So the real download order may be slightly adjusted in order to take advantage of short chances for small file.

\section{G.About Energy Consumption and Replenishment}

If there is a continuous spare period with duration longer than the threshold, a sun-pointing operation will be scheduled in that period.

\section{H.About Decision during Looking Ahead}

Whether a current operation will be accepted using the look-ahead strategy is decided by the following two points.

In fact, the rule-based heuristic method mentioned here may fit quite well for using on board, since it is simple and efficient with less calculation resources needed, and it is adaptive to the inside dynamic feature of the problem.

\section{Re-PlanNing ON BOARD}

The initial ground-generated plan is based on many resource models and may not be very precise. So, re-planning on board will use newly collected information about all the resource status and weather conditions to check the initial plan's feasibility before transforming it into commands for execution. If there are conflicts, re-planning will try to resolve them using simple methods such as cancelling an operation or sliding an operation within its time window.

The re-planning methods are rather simple for two reasons. First, computing capability on board is limited, and there is not much time available for revising the initial plan and transforming it into commands before execution time. Second, from a management aspect, significant changes are not anticipant, since other department on the ground may have already made their own plan in accordance with the initial ground-generated plan, like work shifts schedule at the ground stations. They don't want the plan to be changing, but they even have no chance to get to know the changes under current 
communication conditions. So, the simple methods employed will impose relatively less impact on other ground department.

\section{APPLICATION}

The planning and scheduling process and methods proposed in this paper are designed primarily for a specific AEOS. Since the satellite has some individual features, we don't have benchmark problems to have some tests and comparisons. Practically, the main purpose of this paper is not to provide sophisticated algorithms, but a framework and processes as a whole. Our results have been applied in some simulation cases to assist design optimization of the satellite.

Basic structure of the simulation system is demonstrated in Figure 3. At the top of the graph, there are four primary modules simulating the on-board satellite sub-systems. These are the resource models used to provide real-time satellite status information for planning and scheduling. What need to be noted is that dynamic re-planning is placed within the traditional satellite housekeeping module to simulate the on-board autonomy. The four modules are connected by a CAN bus to simulate the satellite. They are also connected to other modules with an Ethernet, including simulation control, demonstration, planning and scheduling, orbit dynamics and a database/file server. The Planning and scheduling module together with orbit dynamics module are used to simulate the main on-ground decision process. Based on these decisions, simulation control module is in responsible for running the whole simulation.

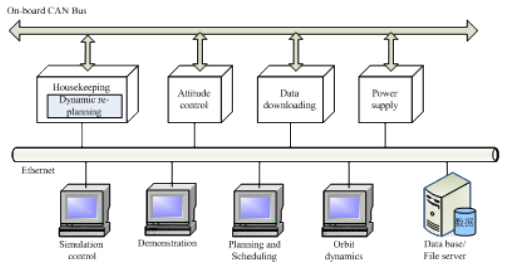

FIGURE III. BASIC STRUCTURE OF THE SIMULATION SYSTEM.

We did some experiments based on the simulation systems. The AEOS orbit parameters at time 2012-07-26 00:00:00 is shown in Table 1 and key parameters of the on-board instruments were list in Table 2 . We chose five ground stations as the available ones which are all inside China.

TABLE I .SATELLITE ORBIT PARAMETERS

\begin{tabular}{|c|c|c|c|c|}
\hline $\begin{array}{c}\text { Semimajor } \\
\text { Axis a(m) }\end{array}$ & $\begin{array}{c}\text { Eccentri } \\
\text { city } \\
\text { e }\end{array}$ & $\begin{array}{c}\text { Inclina } \\
\text { tion } \\
\text { I(d) }\end{array}$ & $\begin{array}{c}\text { RAAN } \\
\boldsymbol{\Omega} \text { (d) }\end{array}$ & $\begin{array}{c}\text { Arg. } \\
\text { of } \\
\text { Periap } \\
\text { sis } \boldsymbol{\omega}\end{array}$ \\
\hline 7051203 & $\begin{array}{c}0.00234 \\
4\end{array}$ & $\begin{array}{c}97.3086 \\
88\end{array}$ & 249.784 & 0 \\
\hline
\end{tabular}

TABLE II .KEY PARAMETERS OF ON-BOARD INSTRUMENT.

\begin{tabular}{|c|c|c|c|c|}
\hline $\begin{array}{c}\text { Max } \\
\text { Roll } \\
\text { Angle } \\
\text { (degree } \\
\text { ) }\end{array}$ & $\begin{array}{c}\text { Max Pitch } \\
\text { Angle } \\
\text { (degree) }\end{array}$ & $\begin{array}{c}\text { Best } \\
\text { ground } \\
\text { resolutio } \\
\text { n (m) }\end{array}$ & $\begin{array}{c}\text { Battery } \\
\text { Capacity } \\
\text { (relative } \\
\text { value) }\end{array}$ & $\begin{array}{c}\text { SSR } \\
\text { Cap } \\
\text { acity } \\
\text { (TB) }\end{array}$ \\
\hline 45 & 45 & 5 & 100 & 1 \\
\hline
\end{tabular}

Scenarios used are randomly generated which cover different numbers of customer requests, and different distribution of ground targets. Targets in instance 2 and 3 are intensively distributed, whereas they are randomly distributed in other instances. Table 3 gives the key parameters of 6 typical scenarios, in which $\mathrm{R}$ denotes the number of customer requests, $S$ denotes the number of strips, $M$ denotes the number of jointing strips requests, $\mathrm{V}$ denotes the sum values of all the requests. Here each request has a value between 1 and 10 which was assigned by the ground decision maker according to emergency and importance of the request.

TABLE III .PARAMETERS OF INSTANCES.

\begin{tabular}{ccccc}
\hline Instances & $\mathbf{R}$ & $\mathbf{S}$ & $\mathbf{M}$ & $\mathbf{V}$ \\
\hline 1 & 47 & 122 & 3 & 254 \\
2 & 46 & 122 & 4 & 295 \\
3 & 42 & 112 & 3 & 278 \\
4 & 46 & 101 & 4 & 297 \\
5 & 200 & 480 & 70 & 1137 \\
6 & 300 & 734 & 150 & 1855
\end{tabular}

Table 4 shows the ground planning results in a 24 hours horizon, in which Profit denotes the total revenue of all completed requests. Time denotes the duration needed for the algorithm to generate a result in second. $\mathrm{P}$ denotes the number of completed requests. Actions denotes the number of all the key operations and auxiliary operations in the final plan. For computing, we use a computer with a P4 CPU of $3.0 \mathrm{GHz}$ and 2G RAM.

TABLE IV .PLANNING RESULTS OF 24 HOURS.

\begin{tabular}{ccccc}
\hline Instances & Profit & Time(s) & P & Actions \\
\hline 1 & 261 & 24 & 46 & 384 \\
2 & 169 & 19 & 29 & 249 \\
3 & 179 & 19 & 27 & 249 \\
4 & 297 & 18 & 46 & 372 \\
5 & 1009 & 81 & 180 & 1263 \\
6 & 1396 & 126 & 239 & 1633
\end{tabular}

Table 5 shows the results of on-board decision, in which AT denotes the average time consumed for re-planning in seconds, NC denotes the times of re-planning triggered in each 24 hours planning horizon, VP denotes the variant in total revenue finally got. The computer used is also with a P4 CPU of 3.0 $\mathrm{GHz}$ and 2G RAM.

TABLE V .PLANNING RESULTS OF 24 HOURS.

\begin{tabular}{llll}
\hline Instances & AT(s) & NC & VP \\
\hline 1 & 5.2 & 15 & -23 \\
2 & 5.1 & 18 & -50 \\
3 & 4.9 & 14 & -37 \\
4 & 5.0 & 16 & -25 \\
5 & 6.5 & 28 & -75 \\
6 & 6.7 & 30 & -82 \\
\hline
\end{tabular}

As table 4 shows that there are relatively more non-completed requests in instance 2 and 3, because targets are too intense and the satellite is still not agile enough to maneuver so quickly. For instance 5 and 6 , data collected for some targets could not be downloaded within the planning horizon since the satellite had flied out of the range of all the ground stations. It 
also shows that there are many auxiliary operations added in the plan to complete the requests. That is just the planning feature of our problem which is different from common scheduling problems.

From the results, it can be seen that the look-ahead algorithm and revising algorithm are both efficient in computing time. For all these scenarios, computing times used is almost all within tens of seconds for look-ahead algorithm and always several seconds for re-planning algorithm.

From Table 5, we can also find that there are frequent status deviations that would trigger the re-planning. Since our simple re-planning algorithm mainly aims to solve conflicts but not to insert new targets, the variant in revenue is always negative, meaning that some requests are cancelled. But in fact, without the on-board re-planning, such deviations may cause damage to the satellite health or cause more useless data.

Also the simulation system is used to help designing the satellite by varying some key parameters to see what will happen on the application effects. In fact, it shows that increasing agility will greatly improve the satellite's ability to satisfy customer requests as 4 times as much.

Such experiments and applications also show that the planning and scheduling framework and algorithms provided in this paper are reasonable in a certain sense.

\section{CONCLUSION}

This paper primarily provides a planning and scheduling framework for a specific AEOS which has some individual features. Mostly ascribing to insufficient communication time windows and limited on-board computing capabilities, decision processes are distributed both on ground and on board. The on-ground part is more complicated and uses a heuristic look-ahead algorithm, whereas the on-board part is quite simple and uses some rules. Those ideas and algorithms are realized within a simulation system. Results of some experiments show that the framework and methods are reasonable and feasible.

In fact, since there are more and more powerful ground computing resources that can be used, ground decision part may use more sophisticated algorithms that generate better solutions. We are trying to develop other techniques for this problem, like constraint reasoning and meta-heuristics that are combined together to improve the computing quality and efficiency. For the on-board decision part, we will try to consider more kinds of changes, especially emergency tasks, with an assumption that the satellite will have more environment detection instruments and become more powerful in computing. Also, we think our work could be extended to more generalized problems with combinatory planning and scheduling features.

\section{REFERENCES}

[1] M. Lemaître, et al., "Selecting and scheduling observations of agile satellites," Aerospace Science and Technology, vol. 6, pp. 367-381, 2002.

[2] M. Vasquez, J. Hao, "A Logic-Constrained Knapsack Formulation and a Tabu Algorithm for the daily photograph scheduling of an earth observation satellite," Computational Optimization and Applications, vol. 20, pp. 137-157, 2001.

[3] G. Beaumet, "Continuous Planning for the Control of an Autonomous Agile Satellite,"2006. 